\title{
Modelos teóricos de discapacidad: un seguimiento del desarrollo histórico del concepto de discapacidad en las últimas cinco décadas
}

\author{
Theoretical models of disability: tracing the historical development of \\ disability concept in last five decades
}

\begin{abstract}
Resumen
El concepto de discapacidad ha sido extensamente debatido en las últimas cinco décadas, dando fruto a múltiples definiciones y a la proliferación de modelos teóricos que tratan de explicar la discapacidad. Pero a pesar de tal abundancia de estudios, parece que seguimos siendo incapaces de explicar la discapacidad siguiendo un único modelo o definición. En este artículo ofrecemos un breve estudio diacrónico y comparativo de los principales modelos teóricos de la discapacidad, resaltando beneficios y aspectos a mejorar. El propósito último del artículo es subrayar la idea de que la discapacidad es un concepto amplio y en constante evolución.

\section{Palabras clave}

Discapacidad, modelos teóricos, modelo médico, modelo social, modelo minoritario.
\end{abstract}

\begin{abstract}
The concept of disability has been extensively debated over the last five decades, resulting in multiple definitions and the proliferation of theoretical models that attempt to explain disability. But in spite of such an abundance of studies, it seems that we are still unable to explain disability following a single model or definition. In this article, we offer a brief diachronic and comparative study of the main theoretical models of disability, highlighting benefits and aspects to be improved. The ultimate purpose of the article is to underline the idea that disability is a broad and constantly evolving concept.
\end{abstract}

\section{Keywords}

Disability, theoretical models, medical model, social model, minority model.

\author{
María Esther Pérez Dalmeda \\ $<$ perezdalmeda@gmail.com>
}

Oslo Metropolitan University. Noruega

\section{Gagan Chhabra}

<gagan.chhabra@hioa.no>

Oslo Metropolitan University. Noruega

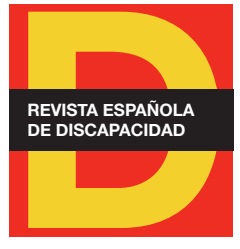

Para citar:

Pérez, M. E. y Chhabra, G. (2019): "Modelos teóricos de discapacidad: un seguimiento del desarrollo histórico del concepto de discapacidad en las últimas cinco décadas". Revista Española de Discapacidad, 7 (I): 7-27.

Doi: <https://doi.org/10.5569/23405104.07.01.01>

Fecha de recepción: 17-01-2018 Fecha de aceptación: 14-02-2019 


\section{Introducción}

El concepto de discapacidad ha sido y sigue siendo extensamente debatido tanto en círculos académicos, como en el terreno político o en organizaciones de personas con discapacidad. En las últimas cuatro décadas los movimientos internacionales de personas con discapacidad han influenciado a organizaciones como las Naciones Unidas, la Organización Internacional del Trabajo y la Organización Mundial de la Salud para que reconocieran la importancia de proporcionar definiciones sobre el concepto de discapacidad (Priestley, 2001). Desde que la Asamblea General de las Naciones Unidas estableciera su primera Declaración de los Derechos de los Impedidos en 1975, el discurso internacional sobre discapacidad dejó de ser un concepto marginal para posicionarse en el centro de los debates. Después, las Naciones Unidas declararon el Año Internacional de los Impedidos en 1981, comenzando así un programa mundial de acción para las personas con discapacidad (Priestley, 2001). Más tarde, en 1985, las personas con discapacidad fueron incluidas por primera vez en la Declaración Universal de Derechos Humanos. Estas acciones de las Naciones Unidas iniciaron el debate sobre las barreras y pusieron de manifiesto el problema de discriminación que sufren las personas con discapacidad (Grue, 2010). Además, con el fin de popularizar el concepto de discapacidad, las Naciones Unidas establecieron el Decenio de las Naciones Unidas para los Impedidos desde 1983 hasta 1992, década que culminó con el Día Internacional de las Personas con Discapacidad (fijado el 3 de diciembre). Tras el Decenio de los Impedidos, las Naciones Unidas desarrollaron una estrategia internacional denominada "Hacia una sociedad para todos", con el objetivo de fomentar la inclusión social de las personas con discapacidad (Priestley, 2001). Junto con las Naciones Unidas, la Organización Internacional del Trabajo incluyó la discapacidad en su agenda global en 1983, adoptando la defensa de un tratamiento igualitario para los trabajadores con discapacidad y demandando la integración social en el mercado laboral (International Labour Organization, 1983: art. 4). Todas estas iniciativas, acciones, programas, planes y estrategias llevadas a cabo por organizaciones internacionales han facilitado y fomentado el discurso sobre discapacidad, dando lugar a nuevos modelos teóricos que tienen por objeto, no solo la definición del concepto, sino la mejora de políticas inclusivas que hagan definitiva la integración de las personas con discapacidad en todos los niveles de la vida social.

Recientemente veíamos en la gala de entrega de los premios Goya 2019 cómo el actor galardonado con el Goya al mejor actor revelación -una persona con discapacidad- mencionaba en su discurso de agradecimiento tres palabras candentes en los debates sobre discapacidad: inclusión, diversidad y visibilidad. $Y$ es que también el hombre común participa en el discurso sobre discapacidad, porque parece que "todo el mundo sabe lo que es la discapacidad" (Tøssebro, 2004: 3, traducción propia). Pero la discapacidad es un fenómeno complejo e impreciso de difícil conceptualización (Waldschmidt, 2017), el cual trasciende "clase, edad, género, nacionalidad, riqueza o fronteras" (Goodley, 2017: 19, traducción propia). La discapacidad puede ser explicada de modo distinto dependiendo de factores tales como el país de origen, el género, la edad, las ideologías políticas o creencias religiosas, o la situación socio-económica (Organización Mundial de la Salud, OMS, 2011). Al pensar en discapacidad nos vienen a la cabeza palabras como 'incapacidad', 'desventaja' o 'deficiencia'. Pensamos en un 'impedimento' que restringe para la realización de actividades 'normales'. Pensamos en algo que 'imposibilita'. Desde un punto de vista económico, la discapacidad es asociada a cierto estatus ligado a algún tipo de restricción social o privación material (Gleeson, 1999). Sin olvidar la conceptualización cotidiana de la discapacidad, que es explicada de modo diferente según las diversas experiencias de los más de mil millones de personas con discapacidad en todo el mundo (OMS, 2011: 
11). Esta es la mayor dificultad a la que se enfrentan los modelos teóricos de discapacidad, que para definir la discapacidad no existe un punto de partida concreto basado en una norma estática, física o funcional. Como consecuencia, el consenso internacional continúa viendo la discapacidad desde varios prismas fraccionados. En este artículo, ofrecemos un breve estudio diacrónico y comparativo de los principales modelos teóricos que conceptualizan la discapacidad, con el fin de exponer y aclarar el estado actual del discurso sobre discapacidad en el panorama internacional.

\section{Los modelos teóricos de discapacidad}

Al hablar de modelos teóricos de discapacidad nos referimos a esquemas teóricos que pretenden ofrecerse como instrumentos empleados para captar y examinar de modo diferente el mundo de la persona con discapacidad, así como para generar nuevas hipótesis y ayudar a evaluar el efecto de situaciones que están más allá de nuestra propia esfera de influencia (Llewellyn y Hogan, 2000). En palabras de Mike Oliver: "Ios modelos son la manera de trasladar ideas a la práctica" (citado en Shakespeare, 2010: 268, traducción propia). Además, habría que puntualizar que la función primordial de los modelos teóricos de discapacidad es ofrecer la explicación del fenómeno mediante la referencia a sistemas abstractos y a elementos de conceptualización y de representación (Altman, 2001). Hasta mediados del siglo XX, la discapacidad era abordada desde una perspectiva individualista, que la explicaba como una tragedia personal o como una deficiencia individual. De este modo, la persona con discapacidad debía ajustarse a su entorno o aceptar la medicalización. Como veremos a continuación, los principales modelos teóricos individualistas son el tradicional, moral o religioso y el médico, rehabilitador o individual. A partir de la Segunda Guerra Mundial, los movimientos por los derechos de las personas con discapacidad en Europa y Norteamérica favorecieron el desarrollo de otros modelos teóricos -el modelo social británico y el modelo minoritario norteamericano-, los cuales definen, interpretan y tratan la discapacidad en relación con la sociedad, moviendo el eje de atención de la persona con discapacidad a la sociedad. Es la sociedad la discapacitada, puesto que en sus políticas y contextos socio-culturales prolonga la discriminación de las personas con impedimentos. En las últimas décadas, el paradigma social ha dado lugar a diversas variantes con diferentes matices teórico-filosóficos.

En las siguientes páginas ofreceremos un recorrido por tales modelos teóricos, siguiendo siempre la misma estructura expositiva: haremos una breve descripción del contexto histórico e influencias filosóficas, sociales o de otros modelos si se da el caso; citaremos los autores y teóricos que han desarrollado cada modelo y pasaremos a dar la definición que dicho prisma teórico aporta de la discapacidad y del impedimento. Finalmente, señalaremos los principales beneficios que los estudiosos han resaltado sobre cada modelo, así como las deficiencias o aspectos a mejorar. El objetivo de este artículo es recopilar en un solo documento las principales características de modelos ya clásicos como el médico, el social o el minoritario, junto a modelos de reciente elaboración como el de derechos humanos, el cultural o el modelo español de diversidad funcional. 


\subsection{Modelos individualistas}

\subsubsection{El modelo tradicional, moral, o religioso}

El modelo tradicional, moral o religioso es el más antiguo y está basado en creencias religiosas. En este modelo la discapacidad es vista como un defecto causado por un fallo moral o un pecado (Goodley, 2017), por lo que la discapacidad está ligada a sentimientos de vergüenza. Toda la familia pasa vergüenza porque uno de sus miembros tiene una discapacidad, de modo que las familias se ven forzadas a ocultar a la persona con discapacidad, apartándola de la escuela y excluyéndola de cualquier posibilidad de desempeñar un papel activo en la sociedad (Miles, 2002), siendo la propia persona con discapacidad responsable de su discapacidad. Para las personas con discapacidad este modelo es particularmente opresivo, generando ostracismo social y autodesprecio (Goodley, 2017).

Bajo el modelo tradicional, moral o religioso la discapacidad conlleva dependencia y vulnerabilidad, lo que desemboca en caridad y cura (Lid, 2012), De hecho, como veremos a continuación, este enfoque coincide con el marco teórico del modelo médico, rehabilitador o individual, el cual ha mantenido la idea de que "con las personas con discapacidad había que ser condescendientes, bendecirlas, dejarlas morir por su enfermedad, institucionalizarlas, curarlas o rehabilitarlas" (Grue, 2011: 535, traducción propia). Porque bajo el modelo tradicional, moral o religioso, al igual que bajo el modelo médico, rehabilitador o individual, las personas con discapacidad son consideradas biológica y psicológicamente inferiores en comparación con las personas sin discapacidad, y son vistas como víctimas defectuosas y frágiles, siendo la discapacidad la única causa de su tragedia personal (Oliver, 1990; Shakespeare, 2004).

En definitiva, bajo el prisma del modelo tradicional, moral o religioso, el padecimiento de enfermedades se explicaba a través de fuerzas espirituales, y la discapacidad era vista como el castigo de un comportamiento pecaminoso (Stone, 1984). Será la teoría genética la que explique la causa de enfermedades a través de agentes externos, debilitando así la creencia de la responsabilidad individual y la virtud moral. Así, en el siglo $\mathrm{XX}$, la relevancia de la teoría genética, los avances en la tecnología y diagnóstico, y la fe en la ciencia y la medicina dieron reconocimiento y legitimidad al modelo médico de discapacidad.

\subsubsection{Modelo médico, rehabilitador o individual}

Según el modelo médico, rehabilitador o individual tener una discapacidad es sinónimo de poseer un cuerpo defectuoso, impedimento o deficiencia que condiciona y restringe la experiencia vital de la persona con discapacidad (Ferreira, 2010). La discapacidad es atribuida al individuo y es vista como una variación negativa de la norma biológica. El modelo médico, rehabilitador o individual se basa en la idea de que los problemas y dificultades que sufren las personas con discapacidad están directamente relacionados con su impedimento físico, sensorial o intelectual (Hahn, 1985; Quinn y Degener, 2002).

La discapacidad impide las capacidades funcionales de la persona con discapacidad. De este modo, los enfermos son automáticamente relegados de cualquier expectativa y responsabilidad social (Barnes y Oliver, 1993), lo que conlleva su institucionalización. Este modelo se basa en el diagnóstico clínico y la categorización (Oliver, 1990), concediéndole el papel primordial a los médicos y profesionales (enfermeras, educadores 
especiales, expertos en rehabilitación, etc.), que puedan curar este defecto o enfermedad, o que hagan de las personas con discapacidad lo más normales posibles. El cuadro 1 ofrece una comparación de los dos modelos individuales, el tradicional, moral o religioso y el médico, rehabilitador o individual.

\begin{tabular}{|c|c|c|}
\hline $\begin{array}{l}\text { Comparación de los } \\
\text { modelos individualistas }\end{array}$ & $\begin{array}{l}\text { La discapacidad como condición tradicional, } \\
\text { moral o religiosa }\end{array}$ & $\begin{array}{l}\text { La discapacidad como condición médica, } \\
\text { rehabilitadora o individual }\end{array}$ \\
\hline Significado & $\begin{array}{l}\text { La discapacidad es vista como un defecto } \\
\text { causado por un fallo moral o un pecado. } \\
\text { Incluye el mito de que, cuando un sentido se } \\
\text { ve perjudicado por la discapacidad, otro se } \\
\text { intensifica. }\end{array}$ & $\begin{array}{l}\text { La discapacidad es un problema médico } \\
\text { que reside en el individuo: un defecto o falla } \\
\text { del sistema corporal que es intrínsecamente } \\
\text { anormal y patológico. El impedimento y la } \\
\text { discapacidad se combinan. }\end{array}$ \\
\hline Implicación moral & $\begin{array}{l}\text { Es una vergüenza para la persona con } \\
\text { discapacidad y su familia. La familia debe } \\
\text { soportar la naturaleza inmoral que conlleva } \\
\text { la presencia de un miembro de la familia con } \\
\text { discapacidad. }\end{array}$ & $\begin{array}{l}\text { Rechaza la visión de la discapacidad como } \\
\text { una lesión en el alma, pero puede culpar a la } \\
\text { persona o la familia por los hábitos de atención } \\
\text { médica (por ejemplo, la personalidad tipo A } \\
\text { conduce al ataque cardíaco) y promulga la visión } \\
\text { de la discapacidad como una tragedia personal. }\end{array}$ \\
\hline Ejemplo & 'Dios nos da lo que podemos soportar'. & $\begin{array}{l}\text { Los pacientes se describen clínicamente (por } \\
\text { ejemplo, el paciente sufre de Trisomía, Síndrome } \\
\text { de Down o tiene una lesión en el nivel C4). } \\
\text { La persona con discapacidad es vista como } \\
\text { atípica, anormal y patológica. }\end{array}$ \\
\hline Origen & $\begin{array}{l}\text { Es el modelo más antiguo de todos y sigue } \\
\text { siendo el más frecuente en todo el mundo. }\end{array}$ & $\begin{array}{l}\text { A mediados de } 1800 \text { en adelante. Supone } \\
\text { el origen de la mayoría de las instituciones y } \\
\text { revistas de rehabilitación de los países ricos. }\end{array}$ \\
\hline $\begin{array}{l}\text { Objetivos de } \\
\text { intervención }\end{array}$ & $\begin{array}{l}\text { Espiritual divina o aceptación. Aumento de } \\
\text { la fe y la tolerancia. Encontrar significado y } \\
\text { propósito en la aflicción. }\end{array}$ & $\begin{array}{l}\text { Se espera que los pacientes o clientes utilicen } \\
\text { los servicios ofrecidos por los profesionales } \\
\text { entrenados con la promesa de curación (la } \\
\text { mejora de la condición física en la mayor medida } \\
\text { posible), rehabilitación (el ajuste de la persona } \\
\text { a su condición) o adaptación (ajuste a la vida } \\
\text { como persona con discapacidad. }\end{array}$ \\
\hline Beneficios del modelo & $\begin{array}{l}\text { La aceptación de ser 'elegido' para tener una } \\
\text { discapacidad, sentir una relación con Dios, } \\
\text { tener un mayor sentido de propósito. Algunos } \\
\text { impedimentos se entienden como evidencia } \\
\text { de encarnación espiritual. }\end{array}$ & $\begin{array}{l}\text { Promueve la fe en la intervención médica y } \\
\text { ofrece una etiqueta como explicación. Los } \\
\text { avances médicos y tecnológicos como servicios } \\
\text { clave del estado de bienestar han mejorado las } \\
\text { vidas de las personas con discapacidad. }\end{array}$ \\
\hline Efectos negativos & $\begin{array}{l}\text { Ser excluido de la familia y la comunidad, } \\
\text { sentimiento de vergüenza, tener que } \\
\text { ocultar los síntomas de discapacidad. La } \\
\text { discapacidad expone las vidas pecaminosas } \\
\text { (pasadas y presentes) de la familia. }\end{array}$ & $\begin{array}{l}\text { Paternalismo, patologización y promoción de la } \\
\text { benevolencia. Promueve el empleo de personas } \\
\text { externas y servicios para las personas con } \\
\text { discapacidad, quienes no son incluidas en los } \\
\text { procesos. }\end{array}$ \\
\hline
\end{tabular}

Fuente: adaptación de Oliver (1996), Goodley (2000), Olkin (2001, 2002, 2009), Barnes y Mercer (2003) (Goddley, 2017: 7, traducción propia).

Según se resalta en el cuadro 1 , el modelo médico ha conllevado muy variadas y diversas consecuencias para las personas con discapacidad. Puesto que es un modelo normativo, los profesionales de la salud y los administradores de beneficios sociales siguen empleándolo como herramienta para clasificar, organizar 
y ofrecer cuidados médicos y servicios sociales (Stone, 1984). Otro claro beneficio del modelo médico, rehabilitador o individual "han sido los tratamientos médicos que sin duda han mejorado la calidad de vida de muchas personas con discapacidad, de hecho han salvado muchas vidas" (Linton, 2010: 224-225, traducción propia). Además, el modelo médico hoy en día sigue influenciando las políticas públicas en cuanto a salud y beneficios sociales, porque como subrayara Mike Bury: "la presencia de una enfermedad crónica como factor desencadenante de discapacidad debe subrayar la necesidad de mayor atención a las medidas preventivas, mejor previsión de cuidados y tratamientos médicos, mayor previsión para adaptaciones en el hogar, y la necesidad de apoyo económico o social" (2008: 86, traducción propia).

La principal crítica negativa tanto al modelo tradicional, moral o religioso, como al médico o rehabilitador, según muestra el cuadro 1 , es que ambos modelos promueven un enfoque individualista que reduce el problema de la discapacidad a la tragedia individual, lo que provoca el ostracismo de la persona con discapacidad que será solo tratable a través de intervenciones de caridad o cuidados médicos (Goodley, 2017). De este modo, "la sociedad, al acceder a seguir el modelo médico de discapacidad, consiente en mantener la discapacidad dentro del ámbito de las instituciones médicas, perpetuándolo como un problema personal, tratando la condición y a la persona con la condición, en vez de tratar los procesos y políticas sociales que limitan las vidas de las personas con discapacidad" (Linton, 2010: 225, traducción propia). Otro aspecto negativo del modelo médico, rehabilitador o individual es que se centra en el impacto desfavorable de la discapacidad física o mental, y no en el entorno donde se desarrolla, siendo el impedimento limitador en sí mismo. En palabras de Dan Goodley, los reduccionistas modelos individualistas -el tradicional y el médico- "han fallado pues no han sabido captar la complejidad de la vida humana, la cual es mucho más que sus componentes biológicos" (2017: 9, traducción propia). Como resultado del enfoque excesivamente patológico e individualista del modelo médico -preocupado exclusivamente por el deterioro-, en la década de los 70 se desarrolla un nuevo modelo de discapacidad: el modelo social, que se centra en la distinción entre discapacidad -exclusión social- e impedimento -limitación física-, y la percepción de las personas con discapacidad como parte de la diversidad humana.

\subsection{Modelo social}

\subsubsection{Modelo social británico}

Durante los años 50 y 60, el modelo médico, rehabilitador o individual dominó el discurso sobre discapacidad en todo el mundo. Será en la década de los 70, tanto en Europa como en Estados Unidos y Canadá, cuando las personas con discapacidad comiencen a organizarse en grupos para luchar contra su relegación en residencias e instituciones, contra la exclusión del mercado laboral que les imposibilitaba ganar un sueldo con el que costear su vida, y su consecuente pobreza (Thomas, 2002).

El modelo social británico emergió de la Unión de los Discapacitados Físicos contra la Segregación (Union of Physically Impaired Against Segregation, UPIAS) en Gran Bretaña, y retó la viabilidad del modelo médico con estudiosos como Vic Finkelstein o Colin Barnes y Mike Oliver (Shakespeare, 2004). En oposición al modelo médico o individual que se centraba en el impedimento del individuo y fallaba al explicar las consecuencias que éste podría ocasionarle (Gustavsson, 2004), el modelo social supone un giro radical al distinguir entre 'impedimento' y 'discapacidad' (Bickenbach et al., 1999). Según resalta Tom Shakespeare, en el modelo 
social "el impedimento no es lo mismo que la discapacidad. El primero es individual y privado, la segunda es estructural y pública. Mientras los doctores y profesionales médicos buscan una cura al impedimento, la verdadera prioridad sería aceptar el impedimento y eliminar la discapacidad... El modelo social define discapacidad como una construcción social, una relación entre las personas con discapacidad y la sociedad incapacitada" (Shakespeare, 2010: 268, traducción propia).

Apoyado en teorías materialistas neo-marxistas, el modelo social no atribuye la discapacidad al individuo, sino al entorno, y reivindica un cambio social. Este modelo se sintetiza en tres postulados: 1. Los principales problemas a los que se enfrentan las personas con discapacidad se derivan de las actitudes sociales y no de las limitaciones funcionales; 2. El entorno creado por el hombre está conformado y moldeado por las políticas sociales, y 3. En una sociedad democrática, las políticas sociales representan las actitudes y valores prevalentes en dicha sociedad (Stein, 2007). En contraste con el modelo médico, rehabilitador o individual, el modelo social postula que la persona es discapacitada debido a la arquitectura, las actitudes y las barreras creadas por la sociedad. Los factores externos que limitan a las personas con discapacidad son lo que realmente determina la capacidad funcional del individuo (Stein, 2007). Además, el modelo social rechaza la noción de funcionalidad normal y no ve la discapacidad en conformidad con una noción predefinida de normalidad, y en lo referido a la identificación de qué capacidades son importantes, postula que esto depende y lo decide la persona con discapacidad, y no los médicos o profesionales (Burchardt, 2004).

\subsubsection{Modelo minoritario norteamericano}

Al mismo tiempo, activistas y estudiosos norteamericanos desarrollaron su propio modelo, una interpretación social que denominaron modelo minoritario. Este modelo estuvo claramente influenciado por movimientos de derechos humanos de activistas negros y de gays y lesbianas durante los años 60 y 70, así como por los soldados que volvían de la Guerra de Vietnam (Goodley, 2017), junto con asociaciones tales como los Centros de Vida Independiente de Berkley (California). Estos últimos se basan en la filosofía de la autodeterminación o desarrollo personal y están dirigidos por estudiantes con discapacidad, que postulan que las personas con discapacidad son los que más saben de sus necesidades, quienes deben tomar la iniciativa, individual y comunitariamente, a la hora de decidir y desarrollar la mejor solución para su situación (DeJong, 1979; Brisenden, 1986). El movimiento de Vida independiente lucha por los derechos civiles de las personas con discapacidad, así como por la desmedicalización y la desinstitucionalización, oponiéndose al dominio profesional y a la provisión burocrática de los servicios sociales y su escasez, mientras demanda oportunidades para que las personas con discapacidad desarrollen sus propios servicios en el mercado (Palacios y Romañach, 2006).

De este modo, el modelo minoritario reta la discriminación de las personas con discapacidad y demanda una redefinición cultural en oposición a las "despiadadas teorías individualistas de las sociedades Americana y Canadiense" (Goodley, 2017: 14, traducción propia). El modelo minoritario reivindica el reconocimiento de la humanidad y la diversidad más allá de los estrechos confines del mercado laboral y el consumismo. Combina críticas neomarxistas del capitalismo con teorías de raza y discriminación, para adoptar una comprensión ecléctica de la formación sociocultural de la discapacidad (Shakespeare y Watson, 2001). En definitiva, el modelo minoritario sintetiza el esfuerzo de los grupos minoritarios y reconoce la diferencia que supone la discapacidad, conectándolo con otras minorías étnicas, raciales, etc. El cuadro 2 muestra una comparación de los dos modelos sociales: el británico y el estadounidense. 


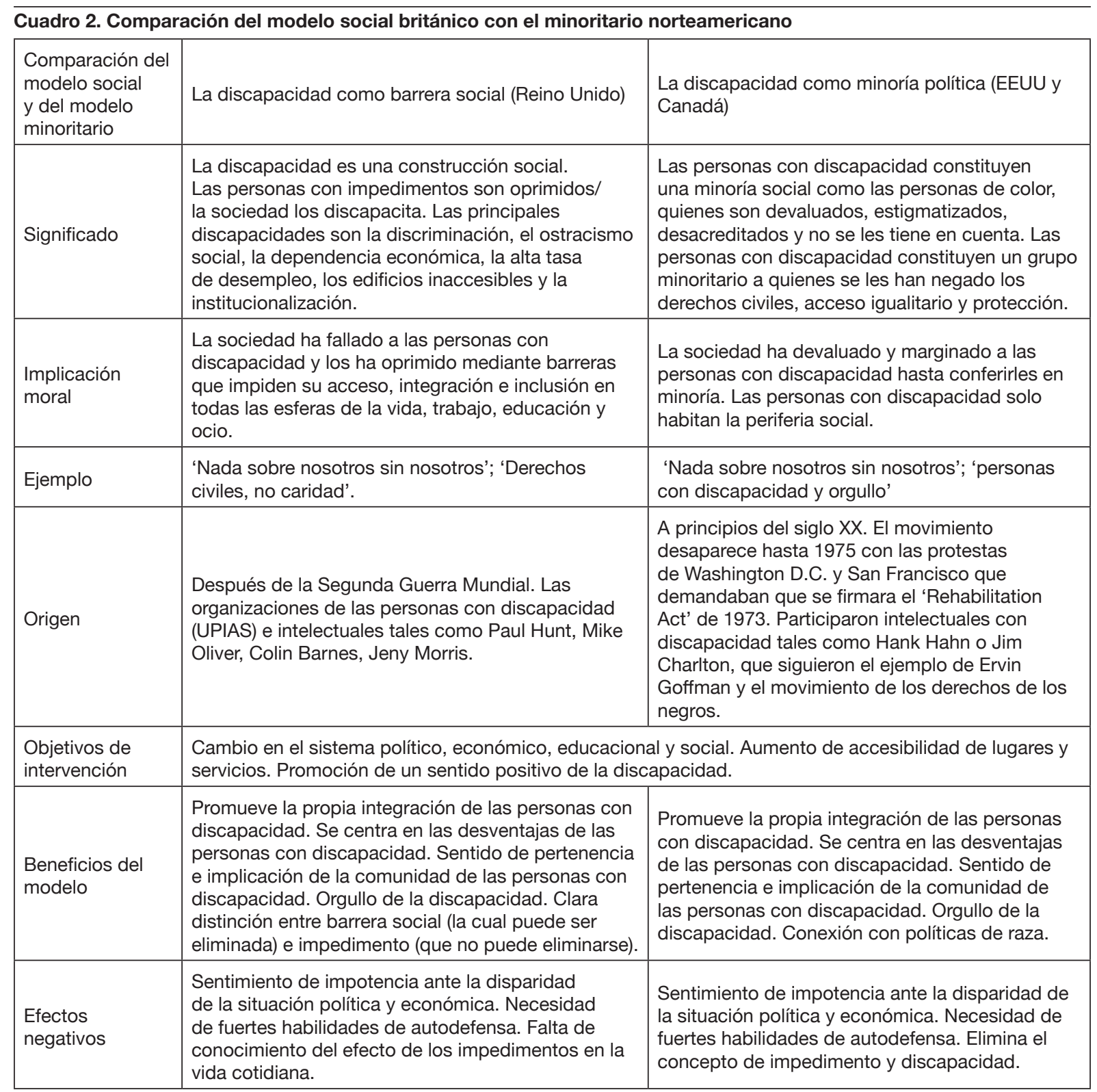

Fuente: adaptación de Oliver (1996), Olkin (2001, 2002, 2009), Gabel (2006), Brandon (2008) (Goodley, 2017: 14: traducción propia).

Tom Shakespeare (2010) destaca tres beneficios fundamentales de los modelos social y minoritario: 1 . Dichos modelos han sido efectivos políticamente: porque han originado movimientos de reivindicación social; 2. Han sido efectivos instrumentalmente, al señalar que para la liberación de las personas con discapacidad se deben eliminar las barreras sociales, y 3 . Han sido efectivos psicológicamente porque han mejorado la autoestima de las personas con discapacidad y han creado un sentimiento positivo en cuanto a la conciencia de identidad. No en vano, tanto en Gran Bretaña como en todo el mundo, muchos activistas se han refugiado en el modelo social para luchar por los derechos y la igualdad en la participación de las personas con discapacidad. 
Pero los modelos social y minoritario también han recibido críticas negativas. Por un lado, siguiendo estos modelos se corre el peligro de reducir la experiencia de ser discapacitado a un fenómeno macrosocial (Stein, 2007). Además, según defienden Tom Shakespeare y Nick Watson (2001), las personas con discapacidad lo son a causa de las barreras sociales, pero también de sus cuerpos. El impedimento y la enfermedad crónica causan efectos directos a las restricciones diarias de la actividad, lo cual constituye la discapacidad. El propio impedimento discapacita (Shakespeare y Watson, 2001), y no tiene sentido negar la relación entre enfermedad crónica/impedimento y discapacidad (Thomas, 2002). De este modo, los detractores del modelo social denuncian la postura de rechazo hacia cualquier concepción de la discapacidad derivada de normas biológicas (Thomas, 2002), pues se corre el riesgo de caer en la noción de que el impedimento no es un problema. Otro aspecto negativo es que al sugerir que las personas son discapacitadas por la sociedad y no por su cuerpo, los modelos social y minoritario pueden ser interpretados como un rechazo a la prevención, rehabilitación o cura del impedimento. También, el modelo social ha descuidado los derechos económicos, sociales y culturales de las personas con discapacidad (Stein, 2007). Además, y aunque es innegable que los modelos social y minoritario han liderado movimientos de protesta de las personas con discapacidad, particularmente en Gran Bretaña y Estados Unidos, según declara Tom Shakespeare (2010), el modelo social ha producido una comprensión un tanto estrecha de la discapacidad.

A pesar de las críticas a las deficiencias metodológicas y teóricas de los modelos social y minoritario, recientemente han resurgido argumentos que los actualizan al incluir derechos humanos y legislativos. Los modelos social y minoritario han proporcionado la base para la elaboración de CIF (OMS, 2001), y han sido incorporados en la Convención sobre Discapacidad de las Naciones Unidas (UN, 2007). Además, las corrientes materialistas que subrayan la opresión de las personas con discapacidad y que luchan por su emancipación lo han enriquecido con estudios lingüísticos o culturales (Gustavsson, 2004), por lo que no se pude negar la influencia y beneficios de los modelos social y minoritario en el discurso internacional sobre discapacidad.

\subsubsection{El modelo biopsicosocial o CIF}

En 1980, influida aún por el modelo médico, la Organización Mundial de la Salud (OMS) aprobó la publicación de una herramienta clasificatoria: la Clasificación internacional de deficiencia, discapacidad y minusvalía. En este documento se definía 'deficiencia' como "toda pérdida o anormalidad de una estructura o función psicológica, fisiológica o anatómica" (OMS, 1983: 54); 'discapacidad' era definida como "toda restricción o ausencia (debida a una deficiencia), de la capacidad de realizar una actividad en la forma o dentro del margen considerado normal para el ser humano" (OMS, 1983: 56), y definía 'minusvalía' como "una situación desventajosa para un individuo determinado, consecuencia de una deficiencia o discapacidad que limita o impide el desempeño de un rol que es normal en su caso (en función de su edad, sexo y factores sociales y culturales)” (OMS, 1983: 57). Esta clasificación en tres niveles -'deficiencia', 'discapacidad' y 'minusvalía'recibió críticas por inclinarse hacia el modelo médico, rehabilitador o individual y por estar fuertemente ligada al concepto de enfermedad, tratando de determinar la normalidad física y psicológica para compararla con la deficiencia y la discapacidad (European Commission, 2002).

Para paliar tales críticas, se creó la Clasificación internacional del funcionamiento, la discapacidad y la salud, CIF (OMS, 2001), con la intención de alcanzar un compromiso viable entre el modelo médico o individual y el social. La CIF determina las áreas de las cuales surgen las discapacidades: 'déficit', 'limitación', 'restricción', 
y 'barrera'. El 'déficit' en el funcionamiento sustituye al término 'deficiencia', y es definido como "la pérdida o anormalidad de una parte del cuerpo o de una función fisiológica o mental" (Romañach y Lobato, 2005: 2). Por su parte, las limitaciones en la actividad sustituyen el término discapacidad y se definen como "las dificultades que una persona puede tener en la ejecución de las actividades. Las limitaciones en la actividad pueden clasificarse en distintos grados, según supongan una desviación más o menos importante, en términos de cantidad o calidad, en la manera, extensión o intensidad en que se esperaría la ejecución de la actividad en una persona sin alteración de la salud" (Romañach y Lobato, 2005: 2). La restricción en la participación sustituye al término minusvalía y se define como "Ios problemas que una persona puede experimentar en su implicación en situaciones vitales" (Romañach y Lobato, 2005: 2). La restricción en la participación se determina al comparar la participación de una persona con discapacidad con la participación esperada por una persona sin discapacidad en una cultura o sociedad determinadas. Por último, las 'barreras' son los factores ambientales que condicionan el funcionamiento de la persona con discapacidad y crean su discapacidad. La definición de barrera es la misma que se ofrecía en el modelo social británico, incluyendo barreras arquitectónicas, tecnológicas, de actitudes, políticas, etc.

La CIF ha sido beneficiosa como herramienta teórica para activistas de los derechos de las personas con discapacidad, investigadores y políticos del panorama internacional, quienes han tomado el modelo biopsicosocial como patrón para desarrollar un entendimiento común del concepto de discapacidad. El marco conceptual de la CIF también ha sido adoptado por la Convención de Naciones Unidas sobre los Derechos de las Personas con Discapacidad (UN, 2007). Además, el Informe Mundial de Discapacidad sigue este modelo y define 'discapacidad' como cualquier aspecto negativo de la interacción entre un individuo (con un problema de salud) y los factores contextuales de ese individuo (factores ambientales y personales) (OMS, 2011).

De todos modos, aunque la CIF propone nueva terminología para intentar desplazar el 'problema' de la discapacidad del individuo a la sociedad, la terminología propuesta prolonga las connotaciones negativas asociadas a déficit, limitación, restricción o barrera. En palabras de Agustina Palacios y Javier Romañach: "ninguno de estos términos es positivo, ni neutro, por lo que resultan un vano intento de cambiar una realidad lingüística y conceptual en la que los propios autores del documento de la OMS no exponen una visión neutra o positiva" (2006: 106). Puesto que el intento de la OMS no ha recibido la aprobación internacional, se han seguido desarrollando modelos teóricos de discapacidad, como veremos a continuación.

\subsubsection{Modelo escandinavo o relacional}

Estudiosos tales como Rannveig Traustadóttir, Jan Tøssebro, Kristiana Kristiansen, Halvor Hanisch, Jan Grue, Simo Vehmas, Dora Bjarnason y Kristín Björnsdóttir de Dinamarca, Noruega, Suecia, Finlandia e Islandia, aplican las teorías sociales angloamericanas para desarrollar el modelo escandinavo o relacional (Grue, 2010). En oposición a las reivindicaciones del Movimiento de Vida Independiente, el modelo escandinavo o relacional resaltan la influencia positiva de los servicios sociales y de los profesionales en las vidas de las personas con discapacidad. El modelo escandinavo o relacional es un tipo de modelo social más flexible (Tøssebro, 2004; Shakespeare, 2004). Si en el modelo social se entendía que la discapacidad estaba únicamente causada por el entorno, en el modelo escandinavo o relacional el concepto base es la relación o interacción entre el individuo y el medio donde desarrolla sus actividades. Otra diferencia en cuanto al modelo social es que el modelo escandinavo o relacional no distingue entre discapacidad e impedimento ya que esta distinción no se aplica a las lenguas nórdicas y no es posible su traducción (Goddley, 2017). 
El modelo escandinavo o relacional parte de tres ideas principales: 1. La discapacidad proviene de un desajuste entre la persona y su medio; 2. La discapacidad es situacional o contextual, y 3. La discapacidad es relativa (Goodley, 2017: 18, traducción propia). La persona con discapacidad tiene unas capacidades, aunque puede que no estén a la altura de las demandas de la sociedad. Como resultado de esta relación entre las capacidades del individuo y la demanda del medio social se abre una brecha. Esta brecha crea y perpetua la discapacidad (Tøssebro, 2004). Por lo tanto, la discapacidad es relativa con respecto al medio y situacional con respecto al contexto en el que la persona con discapacidad realiza su actividad.

Uno de los grandes beneficios de este modo de conceptualizar la discapacidad es que trata de encontrar el equilibrio entre las cualidades y capacidades individuales por un lado, y las demandas sociales y barreras socioambientales por el otro (Grue, 2010). Por esta razón, autores británicos como Tom Shakespeare (2014) y Dan Goodley (2017) defienden la aplicabilidad del modelo escandinavo o relacional y lo proponen como marco viable de partida desde el cual estudiar la interacción entre el cuerpo, la mente y el medioambiente, situando en el centro las contribuciones de los servicios y prácticas sociales, definidas estas como instituciones a las cuales se les ha extirpado el poder o control sobre las personas con discapacidad.

Como crítica al modelo escandinavo o relacional cabría destacar el hecho de que "los servicios y derechos sociales escandinavos son los mejores del mundo" (Goodley, 2017: 18, traducción propia), por lo que la influencia de los servicios sociales sin duda será más positiva en un estado del bienestar como el nórdico que en otros lugares. Además, este modelo es menos cercano a las asociaciones de personas con discapacidad, manteniéndose en una esfera más académica y teórica (Goodley, 2017).

\subsubsection{El modelo de derechos humanos}

En su décimo aniversario, y con la participación de 162 países, la Convención Internacional sobre los Derechos de las Personas con Discapacidad alcanzó un quórum de más del 80 \%, estableciéndose como el primer instrumento de derechos humanos (Degener, 2016). El preámbulo de la Convención subraya que "la discapacidad es un concepto en evolución", que está basada y forma parte de la diversidad humana" (United Nations Convention on the Rights of Persons with Disabilities -UN CRPD- Naciones Unidas, 2006: e, traducción propia). La Convención estipula que todas las personas con discapacidad están sujetas a derechos y que la discapacidad no puede ser utilizada como justificación de la negación o restricción de los derechos humanos (UN CRPD, 2007). Tal enfoque reconoce la discapacidad como construcción social producida por la interacción del impedimento con las barreras sociales, fortaleciendo así el cambio de paradigma del modelo médico al modelo social.

Gerard Quinn y Theresia Degener (2002) señalan que la base de los Derechos Humanos es el reconocimiento de la dignidad humana: todo individuo es válido, no por ser útil económicamente, sino por su valor inherente. Partiendo de esta idea, Theresia Degener (2016: 3-14) define y caracteriza el modelo de derechos humanos en contraposición con el modelo social, y destaca seis principios fundamentales: 1. En el modelo de derechos humanos la discapacidad es entendida como un derecho humano que no requiere determinadas condiciones de salud o un estado corporal. El modelo social no aportaba valores o principios morales, sino que establecía las bases para reformas políticas. Por el contrario, la Convención busca "promover, proteger y garantizar el disfrute pleno e igualitario de los derechos humanos y las libertades fundamentales de las personas con discapacidad, así como promover el respeto a su dignidad inherente" (UN CRPD, 2007, art. 
1, traducción propia). 2. Los Derechos Humanos son más que simples leyes antidiscriminatorias. Mientras el modelo social se centraba en las políticas antidiscriminatorias y en las reformas de los derechos civiles, el modelo de derechos humanos incluye además los derechos económicos, sociales y culturales. 3 . El impedimento debe ser reconocido como una variación humana. El modelo de derechos humanos reconoce el dolor y el deterioro de la calidad de vida ligadas al impedimento y demanda que se tengan en cuenta a la hora de elaborar teorías de justicia social. En este sentido, según subraya Theresia Degener, el artículo 3 de la Convención "es una valiosa contribución a la teoría de derechos humanos ya que aclara que el deterioro no debe considerarse como un déficit o como un factor que puede ser perjudicial para la dignidad humana" (Degener, 2016: 8, traducción propia), por lo que el modelo de derechos humanos propone encontrar modos de respetar, apoyar y celebrar tal diversidad. 4. Debe reconocerse la discriminación múltiple y los diferentes estratos de la diversidad. Si el modelo social británico negaba las políticas de identidad, el modelo de derechos humanos deja espacio para la identificación minoritaria y cultural. Las personas con discapacidad son vistas como un grupo minoritario (Snyder y Mitchell, 2010) similar al de las personas negras u otras minorías étnicas (Barnes et al., 1999). Al igual que en el modelo minoritario, la discapacidad es entendida como parte de la diversidad humana. Esta concepción teórica aleja al modelo de derechos humanos del modelo social británico a la vez que lo acerca al modelo minoritario estadounidense. 5. Las políticas de prevención deben ser sensibles a los derechos humanos. Mientras que el modelo social era crítico con respecto a las políticas preventivas, el modelo de derechos humanos ofrece la base para la evaluación de políticas que garanticen la protección de los derechos de las personas con discapacidad, y promuevan y protejan a las personas con discapacidad, estipulando que la prevención de impedimentos es una cuestión de salud (art 25). La Convención defiende la prevención de posibles discapacidades (art. 25 (b)) y demanda acceso igualitario a los servicios sociales y de salud, los cuales deben basarse en derechos a la libertad y dignidad. 6. Pobreza y discapacidad están interrelacionadas, pero el modelo de derechos humanos puede guiar el cambio. Mientras el modelo social analizaba, comprendía y explicaba por qué dos tercios de los mil millones de personas con discapacidad en el mundo viven en relativa pobreza, el modelo de derechos humanos reconoce que la discapacidad fue excluida de las políticas de desarrollo, y demanda cooperación internacional para incluir la discapacidad en tales programas (art. 32).

Como beneficios de este modelo teórico, podemos destacar el hecho de que la Convención subraye la necesidad de tomar medidas para cambiar actitudes y comportamientos que estigmatizan y marginalizan a las personas con discapacidad. Al subrayar la dignidad como característica inherente al ser humano y el impedimento como parte de la diversidad humana, el modelo de derechos humanos propone ajustar leyes y programas para superar barreras y garantizar el ejercicio de los derechos civiles, culturales, económicos y sociales de las personas con discapacidad.

Pero, aunque es innegable que el modelo de derechos humanos es una buena herramienta para debates académicos y la elaboración de políticas internacionales, este modelo sigue presentando la discapacidad como un problema social que puede ser resuelto mediante políticas de accesibilidad y participación (Waldschmidt, 2017). El modelo de derechos humanos, encorsetado en políticas internacionales, olvida los beneficios que la sociología, la imaginería y prácticas culturales y las experiencias personales pueden aportar a la hora de comprender los efectos del impedimento (Ellis, 2015). En este sentido, los defensores del modelo cultural critican que aunque el modelo de derechos humanos entiende el impedimento como parte de la diversidad humana y destaca las dificultades que este impedimento encuentra en su relación con el medioambiente, olvida que el impedimento está mediatizado socialmente, hecho que deriva en identidad de grupo y perspectivas fenome- 
nológicas (Snyder y Mitchell, 2006). Porque "la discapacidad no es solo una condición médica, sino que esa condición médica aporta significado y connotaciones simbólicas” (Shakespeare, 2014: 50, traducción propia), las cuales necesitan ser comprendidas para poder explicar el significado de discapacidad.

\subsubsection{El modelo cultural}

Estudiosos del campo de las Humanidades como Lennard Davis, Rosemarie Garland-Thomson, Robert McRuer, David Mitchell y Sharon Snyder, Margrit Shildrick, Tobin Siebers o Shelley Tremain (Waldschmidt, 2017), proponen el análisis de textos culturales y literarios para comprender el significado de la discapacidad. El modelo cultural parte de la concepción de la discapacidad como identidad social minoritaria, y asume que impedimento y discapacidad son categorías que construyen la cultura y se nutren de la misma (Waldschmidt, 2017: 20, traducción propia). Este modelo propone entender la discapacidad como "un tropo cultural perteneciente a una comunidad histórica, el cual suscita cuestiones sobre la materialización del cuerpo y las formulaciones sociales que se emplean para interpretar diferencias corporales y cognitivas" (Garald-Thomson, 2002, en Goodley 2017: 16, traducción propia). El modelo cultural considera la discapacidad no como una tragedia individual, ni como un efecto de discriminación y exclusión social, sino que cuestiona la normalidad e investiga el resultado de las prácticas de (des)normalización en la categoría social que hemos venido a llamar discapacidad (Pérez Dalmeda, 2017). La mayoría de los defensores del modelo cultural no distinguen entre discapacidad e impedimento, porque defienden que la biología y la cultura se retroalimentan, lo que implica no ver los cuerpos como fallos biológicos, sino como entidades socioculturales (Goodley, 2017).

Anne Waldschmidt (2017: 19-27) destaca cuatro puntos clave a la hora de definir el modelo cultural: 1. Este modelo considera la discapacidad no como una entidad o hecho dado, sino como un discurso o como un proceso, experiencia, situación o evento. 'Impedimento', 'discapacidad' y 'normalidad' son significantes vacíos que hacen referencia a una suma de características físicas, psicológicas y cognitivas, las cuales tienen en común una serie de connotaciones negativas o positivas otorgadas por la sociedad, el saber académico, los medios de comunicación y el discurso cotidiano. Estas categorías dependen de la situación histórica y las estructuras de poder, y están supeditadas por los discursos hegemónicos. 2. El modelo cultural define la discapacidad como una categoría distintiva, la cual se interpreta dentro de un marco dicotómico de diferencias corporales: sano, completo y normal versus enfermo, deficiente y desviado. De este modo, la discapacidad existe dentro de un orden cultural e histórico, y solo cuando y en la medida en que ciertas diferencias pueden distinguirse y considerarse relevantes para la salud. 3. El modelo cultural reivindica la interdependencia entre las subjetividades individuales y colectivas de las personas con y sin discapacidad. Tanto discapacidad como capacidad se relacionan con los órdenes simbólicos prevalentes y las prácticas institucionales creadas para producir normalidad/desvío. Al asumir el carácter constructivista y discursivo de la discapacidad, se asume la contingencia histórica y la relatividad cultural de la inclusión/exclusión, estigmatización/aceptación, así como patrones socioculturales de experiencia e identidad, creación de significado y práctica, poder y resistencia. 4. En lugar de seguir observando a las personas con discapacidad y preguntándoles a qué tipo de problemas se enfrentan y cómo la sociedad debería apoyarles, el enfoque cultural amplia su perspectiva hacia la sociedad y la cultura en general, con el objetivo de comprender las formas dominantes en relación a la salud, normalidad y funcionamiento.

Uno de los beneficios del modelo cultural es que aleja el foco de estudio del cuerpo impedido al relacionar la discapacidad con el discurso y no con lo anormal (Goodley 2011, en Shakespeare, 2014). De hecho, uno 
de los resultados de no centrarse solo en los aspectos negativos de su representación cultural es que las personas con discapacidad "pueden también crear modos alternativos de pensar y representar la discapacidad, modos que reten estereotipos tradicionales y potencien los aspectos positivos y liberalizadores, o al menos desestabilizadores" (Shakespeare, 2014: 51, traducción propia). Otro aspecto beneficioso del modelo cultural es que en su análisis: "explora las actitudes de las personas sin discapacidad y la noción que estas personas tienen del cuerpo capacitado" (Shakespeare, 2014: 52, traducción propia). De este modo, el modelo cultural implica un cambio epistemológico puesto que demanda que los estudios en discapacidad no se centren solo en la discapacidad, sino en su relación con 'normalidad' y en la intersección de ambas categorías. La discapacidad entendida como un tipo de diferencia relacionada con la salud, el funcionamiento, los logros y la belleza, y sus polos opuestos, puede ofrecer conocimiento esencial a cerca del legado, y la evolución de la sociedad y cultura contemporáneas ${ }^{1}$ (Goodley, 2017).

Como críticas negativas al modelo cultural, cabe destacar que en ocasiones los estudios culturales "parecen mucho más interesados en los textos y el discurso que en las vidas de las personas con discapacidad" (Shakespeare, 2014: 53, traducción propia). El modelo cultural corre el riesgo de olvidar la importancia de escuchar las voces de las personas con discapacidad y lo que ellos tienen que decir acerca de sus desventajas. Otra crítica al modelo cultural es que se aleja del mundo real para centrarse en terminologías, usos lingüísticos y palabras. A este respecto, no se puede negar la necesidad de detenerse en estudios lingüísticos o históricos de la discapacidad, pero además se necesita seguir trabajando en la creación de políticas sociales que protejan y garanticen los derechos de las personas con discapacidad (Shakespeare, 2014).

\subsubsection{Modelo de diversidad funcional}

Asociaciones europeas de personas con discapacidad -European Network of Independent Living o European Disability Forum- han centrado su atención en la salud y en el concepto de servicios personalizados promovidos por el movimiento de Vida Independiente de Estados Unidos, para presionar nacional y supranacionalmente y asegurar los derechos de las personas con discapacidad (Halvorsen y Hvinden, 2009). Iniciativas tales como la disponibilidad de asistencia tecnológica, presupuestos personales y beneficios sociales para el usuario/cliente han fortalecido el movimiento de Vida Independiente a través de Europa (Nordic Cooperation on Disability Issues, 2007; EPR, 2013). Siguiendo dicho impulso internacional, en España en 2001 se crea una comunidad virtual denominada Foro de Vida Independiente ${ }^{2}$ (FVI), la cual "se constituye como un espacio reivindicativo y de debate a favor de los derechos humanos de las mujeres y hombres con todo tipo de discapacidad de España" (Romañach y Lobato: 2005: 5). El FVI se crea, además, como una plataforma desde donde debatir y difundir la filosofía del Movimiento de Vida Independiente (Palacios y Romañach, 2006), dando lugar al desarrollo del modelo de diversidad funcional.

El modelo de diversidad funcional se basa en el modelo minoritario norteamericano, pues entiende la discapacidad como parte de la diversidad humana, diversidad que es entendida como fuente de riqueza ( $\mathrm{Pa}-$

1. Recientes estudios en discapacidad apuntan a una concepción posthumanista de la discapacidad (Braidotti, 2013), vista no como una tragedia, sino como posibilidad: "bajo el paradigma posthumano, el cuerpo es necesariamente relacional, fluido y múltiple" (Dolezal, 2017: 60, traducción propia). "De hecho, la unión entre el perro guía y el ser humano; o entre el asistente personal y su cliente, nos exige pensar de nuevo acerca de las viejas nociones humanistas de autonomía, responsabilidad y subjetividad" (Goodley et al., 2014: 355, traducción propia). El cuerpo posthumano es un cuerpo de "posibilidad y capacidad a menudo aumentada, modificada o mejorada por cirugías, genética, prótesis, implantes y tecnologías, las cuales difuminan las líneas entre uno mismo y el otro, humano y animal, hombre y máquina" (Dolezal, 2017: 61, traducción propia).

2. Más información: http://forovidaindependiente.org/. 
lacios y Romañach, 2008), y propone desterrar el concepto de 'capacidad': "La cuestión, entonces, no es la carencia de capacidades, sin el no reconocimiento ni aceptación de ciertos modos particulares, diversos, de desenvolvimiento. En un mundo en el que hemos aprendido a convivir con todo tipo de diversidades (de creencia, de etnia, de cultura, de nacionalidad, de preferencias estéticas, de ideología, de orientaciones sexuales...), hay una particularidad, a la que no hemos querido integrar. La diversidad funcional constituye una más entre las muchas manifestaciones de las infinitas heterogeneidades que caracterizan a la existencia humana" (Ferreira, 2010: 58-59). El modelo de diversidad funcional propone que el eje teórico de la capacidad sea sustituido por el de la dignidad. Al igual que el modelo de derechos humanos, el modelo de diversidad funcional garantiza la dignidad humana.

Siguiendo enfoques de tintes lingüísticos del modelo cultural, Agustina Palacios y Javier Romañach (2006, 2008) proponen un análisis semántico de la palabra 'dignidad' representada en varios textos culturales (jurídicos y bioéticos principalmente) para concluir que la dignidad se articula en dos vertientes: "la primera está relacionada con la igualdad del valor de las vidas de todas las personas y la segunda con la igualdad de derechos de todos los seres humanos" (Palacios y Romañach, 2008: 45). Para hacer realizable los dos tipos de dignidad, el modelo de diversidad funcional propone, al igual que el modelo de derechos humanos, la defensa, difusión e implantación de la Convención Internacional sobre los Derechos de las Personas con Discapacidad de la ONU. A través de la Convención, según Agustina Palacios y Javier Romañach (2008) se hará viable la primera vertiente de dignidad humana. Para hacer realizable la segunda: "Debe desarrollarse una novedosa aproximación bioética que nace del propio colectivo de personas discriminadas por su diversidad funcional, y cuya visión sobre su propia realidad ha sido históricamente ignorada en el ámbito de la bioética, de manera que han sido vistos como seres humanos que sufrían por ser diferentes, en lugar de llegar a entender que el verdadero sufrimiento viene derivado del hecho de ser sistemáticamente discriminados por su diferencia y del desconcierto vital que nace de ver cómo sus vidas siempre han sido percibidas como vidas de diferente valor" (Palacios y Romañach, 2008: 45). Con el fin asegurar la dignidad humana se precisa luchar contra ciertos estereotipos socioculturales que confunden la enfermedad con la diversidad funcional, la autonomía moral con la autonomía funcional, o el mito del sufrimiento. Para ello, el modelo de diversidad funcional propone el empleo de la bioética como herramienta de cambio (Palacios y Romañach, 2006), así como una evolución en la terminología que implique un avance en la ideología. Abandonar la palabra 'minusvalía' de indudable carácter médico y peyorativo, sobrepasar la palabra 'discapacidad', propuesta por el modelo social pero que hace referencia a las capacidades de las personas, para avanzar hacia el binario 'diversidad funcional”.

Uno de los aciertos del modelo de diversidad funcional es que la propuesta de la nomenclatura parte del propio colectivo de personas con discapacidad y no de círculos académicos, teóricos o políticos. Este colectivo decide una nomenclatura positiva 'diversidad funcional' para definir a un grupo de personas que es como el resto, no carece de capacidades, sino que funciona de una manera que no es la habitual. Este modelo, a través de la nueva nomenclatura, pretende resaltar que las personas con diversidad funcional realizan las mismas funciones que los demás, pero de un modo diferente (Ferreira, 2010). Otro claro beneficio es la labor de difusión didáctica y política que el colectivo FVI ha realizado y sigue realizando en España. El FVI ha conseguido que el concepto de vida independiente se incorporara en la Declaración de Madrid, en el artículo 2 de la LIONDAU33 así como en el Plan Nacional de Accesibilidad 2004-2012 del Instituto de Mayores y Servicios Sociales, Imserso ${ }^{4}$.

3. Ley 51/2003 de Igualdad de Oportunidades, no Discriminación y Accesibilidad Universal de las personas con discapacidad. 4. Para más información sobre la historia, evolución y labor del FVI ver Palacios y Romañach, 2006: 60-64. 
Una de las mayores críticas al modelo de diversidad funcional, según señala Miguel Ferreira es que la nomenclatura propuesta "no es un concepto teóricamente fundado ni sociológicamente consistente; de hecho, dicha fundamentación, teóricosociológica, resulta cuando menos complicada" (2010: 59). La palabra 'diversidad' no posee un valor neutro, sino que su significado está jerarquizado según su contexto históricosocial. Del mismo modo, el término 'funcional' crea confusión, puesto que parece hacer relación a la tradición teórica funcionalista (Rodríguez y Ferreira, 2010). Otra crítica teórica es que la nomenclatura propuesta sigue haciendo relación al cuerpo, un cuerpo diferente, comparado a una norma. Ya no de menos valía, o de falta de capacidad, pero sí singular en su funcionamiento, en comparación con un funcionamiento 'normal' (Ferreira, 2010).

\section{Conclusión}

Según se ha evidenciado a lo largo de este artículo, el concepto de discapacidad es complejo y multifacético. Nuestro punto de partida es la idea de que la noción de discapacidad ha evolucionado a lo largo de la historia, desde una concepción estrecha y limitada, médicamente determinada e individualista, hasta una conceptualización más amplia construida social o incluso lingüísticamente. En la actualidad, en la pluralista sociedad moderna, la discapacidad es entendida como una experiencia al mismo tiempo que como una característica propia de la condición humana (UN, 2007). Tras llevar a cabo este recorrido diacrónico por los distintos modelos teóricos de discapacidad surgidos de la amalgama de los modelos médico, social británico y minoritario norteamericano, este artículo cumple el objetivo de resaltar la proliferación de modelos, definiciones y conceptualizaciones de la discapacidad, que ponen de manifiesto la diversidad ideológica en el panorama internacional. En este artículo no nos decantamos por una única definición universal, ni por un gran modelo teórico unificador que abarque y explique el concepto de discapacidad, pero sí queremos resaltar que consideramos imperativo que el discurso internacional sobre discapacidad continúe las comparaciones de políticas internacionales (UN CRPD, 2007, art 32), con el fin de desarrollar una comprensión integral y amplia de la discapacidad.

Una de las conclusiones de este artículo es que todos los modelos teóricos resultantes del impulso propinado por el modelo social y minoritario ofrecen diferentes matices que de modo ecléctico pueden ser empleados para definir y conceptualizar la discapacidad. En este sentido, como subraya Martha Nussbaum (2004) debemos trascender la clasificación binaria de discapacidad/no discapacidad y postular por una categoría universal de discapacidad basada en la personificación y la vulnerabilidad, ya que "todos poseemos cuerpos mortales y en descomposición, todos padecemos necesidades y discapacidades de diversos tipos y en diversos grados" (Nussbaum, 2004: 341, traducción propia). Ya a mediados de los 80, Deborah Stone postulaba la necesidad de liberar la discapacidad como categoría de presiones sociales, políticas, judiciales, médicas y económicas, y proponía que la discapacidad como categoría debía estar en constante expansión, porque "todos estamos en riesgo de discapacidad, desde el momento en que nacemos, e incluso antes" (Stone, 1984: 192, traducción propia). Por eso, nos gustaría resaltar el valor de los estudios en discapacidad, los cuales tienen el potencial para contribuir a la redefinición de la sociedad y la cultura (Wolfe, 2010), puesto que demandan interdisciplinaridad, constante diálogo y experimentación que rete filosófica y éticamente el significado de (dis)capacidad. Según señala la corriente filosófica poshumanista: "la anomalía humana (...) 
continua problematizando la retórica del individualismo liberal, poniendo a prueba la ética de la tolerancia y la fetichización de autonomía y voluntad humana y la participación civil" (Kaplan, 2000 en Wolfe, 2010: 139, traducción propia). Porque como señala Dan Goodley "debemos empezar con la discapacidad pero nunca terminar con ella, La discapacidad es el espacio desde el cual pensar una serie de cuestiones políticas, teóricas y prácticas que son relevantes para todos" (Goodley, 2017: 82, traducción propia). En definitiva, es imperante continuar el dialogo y proseguir estudiando la discapacidad, no solo en beneficio de las personas con discapacidad, sino de toda la sociedad. 


\section{Referencias bibliográficas}

Altman, B. M. (2001): "Disability Definitions, Models, Classification Schemes, and Applications”, en Albrecht, G. L. et al. (eds.): Handbook of Disability Studies. Thousand Oaks, California: Sage Publications.

Barnes, C. et al. (1999): Exploring Disability: A Sociological Introduction. Cambridge: Polity Press.

Barnes, C. y Mercer, G. (2003): Disability: Key Concepts. Cambridge: Polity Press.

Barnes, C. y Oliver, M. (1993): Disability: A Sociological Phenomenon Ignored by Sociologists (en línea). <https:// disability-studies.leeds.ac.uk/wp-content/uploads/sites/40/library/Barnes-soc-phenomenon.pdf>, acceso 20 de diciembre de 2017.

Bickenbach, J. E. et al. (1999): "Models of disablement, universalism and the international classification of impairments, disabilities and hándicaps”. Social Science and Medicine, 48: 1173-1187.

Brandon, T. (2008): "Is being fat the new disability?" En Research Institute of Health and Social Change Annual Conference. Conferencia llevada a cabo en Manchester.

Braidotti, R. (2013): The posthuman. Londres: Polity.

Brisenden, S. (1986): "Independent living and the medical model of disability”. Disability, Handicap and Society, 1 (2): 173-178.

Burchardt, T. (2004): "Capabilities and Disability: The Capabilities Framework and the Social Model of Disability". Disability \& Society, 19 (7): 735-571.

Bury, M. (2008): "Defining and Researching Disability: Challenges and Responses”, en Watson, N. (ed.): Disability: Major Themes in Health and Social Welfare, Vol. III, 57. Londres: Routledge.

Degener, T. (2016): "Disability in Human Rights Context”. Laws, 5 (3): 35 (en línea).

<https://doi.org/10.3390/laws5030035>, acceso 19 de mayo de 2019.

DeJong, G. (1979): "Independent living: from social movement to analytic paradigm”. Archives of Physical Medicine and Rehabilitation, 60 (10): 435-446.

Dolezal, L. (2017): “Representing Posthuman Embodiment: Considering Disability and the Case of Aimee Mullins". Women's Studies, 46 (1): 60-67.

Ellis, K. (2015): Disability and Popular Culture: Focusing Passion, Creating Community and Expressing Defiance. Farnham: Ashgate.

European Commission (2002): Definition of Disability in Europe. A comparative analysis. Londres: Brunel University.

Ferreira, M. A. (2010): “De la minus-valía a la diversidad funcional: un nuevo marco teórico-metodológico”. Política y sociedad, 47 (1): 45-65.

Gabel, S. (2006): "Disability studies and inclusive education: negotiating tensions and integrating research, policy and practice". En Second International City Conference of Disability Studies Studies in Education. Conferencia llevada a cabo en Michigan.

Gleeson, B. (1999): Geographies of Disability. Londres: Routledge. 
Goodley, D. (2017): Disability Studies. Londres: Sage.

Goodley, D. (2000): Self-advocacy in the Lives of People with Learning Difficulties: The Politics of Resilience. Maidenhead: Open University Press.

Goodley, D. et al. (2014): "Posthuman disability studies". Subjectivity. 7: 343-361.

Grue, J. (2011): “Discourse analysis and disability: Some topics and issues”. Discourse \& Society, 22: 532-546.

Grue, J. (2010): "Is there something wrong with society, or is it just me? Social and medical knowledge in a Norwegian anti-discrimination law". Scandinavian Journal of Disability Research, 12: 165-178.

Gustavsson A. (2004): "The role of theory in disability research -springboard or strait- jacket?". Scandinavian Journal of Disability Research, 6 (1): 55-70.

Hahn, H. (1985): "Towards a politics of disability: definitions, disciplines, and policies". The Social Science Journal, 22 (4): 87-105.

Halvorsen, R. y Hvinden, B. (2009): "Nordic Disability Protection Meeting Supranational Equal Treatment Policy - A Boost for the Human Rights of Persons With Disabilities?", en Aessen, H. S. et al. (eds.): Human Rights, Dignity and Autonomy in Health Care and Social Services: Nordic Perspectives. Antwern: Intersentaia.

International Labour Organization (ILO) (1983): Vocational Rehabilitation and Employment of Disabled Persons (en línea). <http://www.ilo.org/public/english/standards/relm/ilc/ilc86/r-iii1ba.htm>, acceso 20 de diciembre de 2017.

Llewellyn, A. y Hogan, K. (2000): “The Use and Abuse of Models of Disability”. Disability \& Society, 15 (1): $157-165$.

Lid, I. M. (2012): “Disability as a human condition discussed in a theological perspective”. Diaconia, 3: 149-171.

Linton, S. (2010): “Reassigning Meaning”, en Davis, L. J. (ed.): The Disability Studies Reader (3rd edition). Nueva York: Routledge.

Miles, M. (2002): “Disability in an Eastern Religious Context”. Journal of Religion, Disability and Health, 6: 35-37.

Naciones Unidas (2006): Convención sobre los Derechos de las Personas con Discapacidad. Nueva York: ONU.

Nordic Cooperation on Disability Issues (2007): Provision of Assistive Technology in the Nordic Countries. Estocolmo: Nordic Cooperation on Disability Issues.

Nussbaum, M. (2004): Hiding from humanity: Disgust, shame and the law. New Jersey: Princeton University Press.

Oliver, M. (1996): Understanding disability: from theory to practice. Basingstoke: Macmillan.

Oliver, M. (1990): The Politics of Disablement. Basingstoke: Macmillan.

Olkin, R. (2009): Women with Physical Disabilities Who Want to Leave Their Partners: A Feminist and DisabilityAffirmative Perspective. San Francisco: California School of Professional Psychology and Through the Looking Glass, Co.

Olkin, R. (2002): "Could you hold the door for me? Including disability in diversity". Cultural Diversity \& Ethnic Minority Psychology, 8 (2): 130-137.

Olkin, R. (2001): “Disability-affirmative therapy”. Spinal Cord Injury Psychosocial Process, 14 (1): 12-23.

Organización Mundial de la Salud (OMS) (2011): World Report on Disability (en línea). <https://www.who.int/disabilities/world_report/2011/report.pdf>, acceso 3 de febrero de 2019. 
Organización Mundial de la Salud (OMS) (2001): Clasificación Internacional del Funcionamiento de la Discapacidad y de la Salud: CIF. Madrid: Ministerio de Trabajo y Asuntos Sociales, Instituto de Migraciones y Servicios Sociales.

Organización Mundial de la Salud (OMS) (1983): Clasificación internacional de deficiencias, discapacidades y minusvalías. Madrid: Instituto Nacional de Servicios Sociales.

Palacios, A. y Romañach, J. (2008): "El modelo de la diversidad: una nueva vision de la bioetica desde la perspectiva de las personas con diversidad funcional (discapacidad)". Intersticios: Revista sociológica de pensamiento crítico, 2 (2): 37-47.

Palacios, A. y Romañach, J. (2006): El modelo de la diversidad. La bioética y los derechos humanos como herramientas para alcanzar la plena dignidad en la diversidad funcional. La Coruña: Ediciones diversitas.

Pérez, M. E. (2017): “Anne Waldschmidt, Hanjo Berressem y Moritz Ingwersen (eds.) (2017): Culture - Theory Disability: Encounters between Disability Studies and Cultural Studies. Bielefeld: Transcript Verlag". Revista Española de Discapacidad (REDIS), 5 (2): 239-242.

Priestley, M. (2001): Disability and the Life Course Global Perspectives. Londres: Cambridge University Press.

Quinn, G. y Degener, T. (2002): Human Rights and Disability: The Current Use and Future Potential of United Nations Human Rights instruments in the Context of Disability. Nueva York: United Nations.

Rodríguez, S. y Ferreira M. A. V. (2010): "Diversidad Funcional: sobre lo normal y lo patológico en torno a la condición social de la discapacidad”. Cuaderno de Relaciones Laborales, 28 (1): 151-172 (en línea). <https:// revistas.ucm.es/index.php/CRLA/article/view/33363>, acceso 19 de mayo de 2019.

Romañach, J. y Lobato, M. (2005). "Diversidad funcional, nuevo término para la lucha por la dignidad en la diversidad del ser humano” (en línea). <http://forovidaindependiente.org/diversidad-funcional-nuevo-termino-parala-lucha-por-la-dignidad-en-la-diversidad-del-ser-humano/>, acceso 30 de mayo de 2018.

Shakespeare, T. (2014): Disability Rights and Wrongs Revisited. Nueva York: Routledge.

Shakespeare, T. (2010): “The Social Model of Disability”, en Davis, L. J. (ed.): The Disability Studies Reader (3rd edition). Nueva York: Routledge.

Shakespeare, T. (2004): "Social models of disability and other life strategies". Scandinavian Journal of Disability Research, 6 (1): 8-21.

Shakespeare, T. y Watson, N. (2001): “The Social Model of Disability: An Outdated Ideology?". Research in Social Science and Disability, 2: 9-28.

Snyder, S. L. y Mitchell, D. T. (2010): "Introduction: Ablenationalism and the Geo-Politics of Disability". Journal of Literary \& Cultural Disability Studies, 4 (2): 113-125.

Snyder, S. L. y Mitchell, D. T. (2006): Cultural Locations of Disability. Chicago: University of Chicago Press.

Stein, M, A. (2007): “Disability Human Rights”. California Law Review, 95 (1): 75-121.

Stone, D. (1984): The Disabled State. Temple University Press: Philadelphia.

Thomas, C. (2002): “Disability Theory: Key Ideas, Issues and Thinkers”, en Barnes, C. et al. (eds.): Disability Studies Today. Cambridge: Polity Press.

Tøssebro, J. (2004): "Introduction to the special issue: Understanding disability". Scandinavian Journal of Disability Research, 6 (1): 3-7. 
Modelos teóricos de discapacidad: un seguimiento del desarrollo histórico del concepto de

Waldschmidt, A. (2O17): "Disability goes cultural: The Cultural Model of Disability as an Analytical Tool", en Waldschmidt, A. et al. (eds.): Culture-Theory-Disability: Encounters between Disability Studies and Cultural Studies. Bielefeld: Transcript Verlag.

Wolfe, C. (2010): What is Posthumanism? Mineapolis: University of Minnesota Press. 